\title{
The UK Paediatric Ocular Trauma Study 3 (POTS3): clinical features and initial management of injuries
}

This article was published in the following Dove Press journal: Clinical Ophthalmology

\author{
Robert J Barry ${ }^{1,2}$ \\ Freda $\mathrm{Sii}^{1,3}$ \\ Alice Bruynseels' \\ Joseph Abbott ${ }^{4}$ \\ Richard J Blanch ${ }^{1,2,5}$ \\ Caroline J MacEwen ${ }^{6}$ \\ Peter Shah ${ }^{1,3,7,8}$ \\ 'Department of Ophthalmology, Queen \\ Elizabeth Hospital Birmingham, \\ University Hospitals Birmingham NHS \\ Foundation Trust, Birmingham, UK; \\ ${ }^{2}$ Neuroscience and Ophthalmology, \\ Institute of Inflammation and Ageing, \\ University of Birmingham, Birmingham, \\ UK; ${ }^{3}$ Birmingham Institute for Glaucoma \\ Research, Institute of Translational \\ Medicine, University Hospitals \\ Birmingham NHS Foundation Trust, \\ Birmingham, UK; ${ }^{4}$ Department of \\ Ophthalmology, Birmingham Children's \\ Hospital NHS Foundation Trust, \\ Birmingham, UK; ${ }^{5}$ Academic Department \\ of Military Surgery and Trauma, Royal \\ Centre for Defence Medicine, Dundee, \\ UK; ' ${ }^{6}$ Department of Ophthalmology, \\ Ninewells Hospital and Medical School, \\ Dundee, UK; ${ }^{7}$ University College \\ London, London, UK; ${ }^{8}$ Centre for Health \\ and Social Care Improvement, School of \\ Health and Wellbeing, University of \\ Wolverhampton, Wolverhampton, UK
}

Correspondence: Freda Sii

Department of Ophthalmology, Queen

Elizabeth Hospital Birmingham, University

Hospital, Birmingham NHS Foundation

Trust, Mindelsohn Way, Edgbaston,

Birmingham BI5 2WB, UK

Email sii.freda@gmail.com
Purpose: Worldwide, as many as 6 million children annually sustain ocular trauma, with up to a quarter of a million children requiring hospitalization. Management of pediatric ocular trauma differs from that in adults, both in terms of initial assessment and acute intervention, with significant variation in practice between different centers. Patterns of healing and long-term outcomes are also very different for children compared to adults. In order to develop effective protocols for management, it is first necessary to understand current trends in presentation and treatment.

Methods: We conducted a prospective, observational study of pediatric ocular trauma presenting to UK-based ophthalmologists over a one-year period; reporting cards were distributed by the British Ophthalmic Surveillance Unit, and clinicians were asked to report cases of acute orbital and ocular trauma in children aged 16 years or less requiring inpatient or day-case admission. A validated, standardized questionnaire was sent to reporting ophthalmologists to collect data on clinical features and initial management of injury.

Results: Eighty-six episodes of pediatric ocular trauma were reported. Trauma involving the globe was reported in 66/86 patients (76.7\%), of which 40/66 (60.1\%) were open-globe. Trauma to the anterior segment was reported in $57 / 86(66.3 \%)$, and posterior segment in 23/86 patients (26.7\%). Twenty-five of 86 (29.1\%) patients sustained severe trauma defined as having bestcorrected visual acuity worse than $6 / 60$ Snellen (incidence 0.19 per 100,000 population).

Conclusions: There has been no improvement in the incidence or severity of pediatric ocular injury rates over the past 25 years. Eye-care providers must be able to provide the necessary services for assessment and management of severe pediatric ocular trauma in the emergency setting.

Keywords: childhood eye injury, incidence, management, penetrating eye injury, perforating eye injury, presentation, prevention

\section{Background}

Pediatric ocular trauma is common and can profoundly affect a child's development and adult life. Worldwide, a quarter of a million children require hospitalization for a serious ocular injury every year. ${ }^{1}$ As many as 6 million children annually sustain some form of ocular trauma. ${ }^{2}$ Long-term morbidity from ocular trauma in both children and adults is significant; 3.9 million have bilateral visual loss and over 18 million unilateral visual loss. ${ }^{3}$ Visual impairment in children has a disproportionately greater impact upon quality of life than sight loss in older age. ${ }^{4}$

Pediatric ocular trauma is distinct from adult ocular trauma particularly in the domains of assessment and management. In common with adult civilian trauma, injury 
is most commonly uniocular, but this will often result in amblyopia among children 7 years of age or younger. ${ }^{5}$ Furthermore, an injured child is often a poor historian and a thorough assessment can be challenging. Limited evidence is available on presenting clinical features in pediatric ocular trauma, but injuries are characterized by anterior segment pathology: corneal/scleral laceration, iris prolapse, and lens abnormalities. ${ }^{6}$ Studies have demonstrated that children requiring hospitalization for ocular injury are most likely to be male, undergoing treatment for an open wound of the ocular adnexa, with an average length of stay of $2-4$ days. ${ }^{7-10}$ The management of pediatric ocular trauma varies dramatically across UK centers and worldwide, with rates of surgical management ranging from $7.4 \%$ to $59 \%$ depending on the mechanism of injury and presenting features. ${ }^{8,9,11}$

Fortunately, pediatric ocular trauma is thought to be preventable in as many as $90 \%$ of the cases through improved recognition of risk factors for ocular injury and targeted intervention. ${ }^{12-15}$ In order to implement such targeted intervention, it is first necessary to understand the etiology of ocular trauma within the pediatric population, so that preventative measures can be directed toward those children most at risk of injury. Historically, risk factors for pediatric ocular trauma have not been well characterized, and it is estimated that $30 \%$ of all ocular trauma still occurs in childhood. ${ }^{12}$

The UK Paediatric Ocular Trauma Study (POTS) has been developed and implemented to address this knowledge deficit, with the aim of reducing the incidence and improving outcomes of pediatric ocular trauma worldwide. Standardized data collection proformas have been validated and distributed through the British Ophthalmic Surveillance Unit (BOSU), ${ }^{16}$ with preliminary data describing demographics and mechanism of injuries previously published. ${ }^{17}$ To date, this series has demonstrated that boys are more than twice as likely as girls to sustain ocular trauma, with injuries predominantly occurring at home, during play and often involving a sharp implement. Simple preventative strategies have been suggested based on these observations.

Having described demographics and mechanism of injury ${ }^{17}$ we now wish to present clinical features and initial management of pediatric ocular trauma in this population.

\section{Materials and methods}

The UK POTS incident questionnaire was developed to collect data on the demographics, incidence, and mechanism, presenting features and initial management of serious pediatric ocular trauma. Development and validation of this data collection proforma have previously been reported in detail, ${ }^{16}$ and preliminary results comprising patient demographics, incidence of pediatric trauma, and mechanism of injury have been published. ${ }^{17}$

Questionnaires were sent to UK-based ophthalmologists over a 12-month period between 1st June 2014 and 30th May 2015. The study was conducted using the BOSU reporting card scheme; at the end of each month, cards were sent to all UK consultant ophthalmologists in the BOSU database. Clinicians were asked to report any new cases of ocular trauma in children aged 16 years or under presenting in the preceding month that required hospital admission for observation, treatment or surgery. Ophthalmologists were requested to include both patients referred to them, and patients they referred on to other ophthalmologists; this was cross-referenced to ensure each case was recorded only once.

Reporting cards were returned to BOSU, who informed the principal investigator (FS) about each case. The investigator then contacted the reporting ophthalmologist directly. The reporting clinicians were requested to fill out and return the incident data collection questionnaire to the POTS team. A follow-up request was sent to reporting ophthalmologists if no response was received within three months of the initial request. Specific strategies that were used to increase the response rate during the study period have previously been described in detail. ${ }^{16}$

Data were entered into a Microsoft Excel spreadsheet for initial coding and calculation of descriptive statistics. Visual acuity is reported as Snellen but was converted to $\operatorname{logMAR}$ equivalent for statistical analysis. Statistical analysis was performed using SPSS (Version 25, IBM, New York, USA); associations between presenting features and demographics were assessed using a generalized linear model, with categories collapsed where necessary to improve model fit. Results were considered statistically significant when $p<0.05$. $P$-values were corrected for multiple comparisons using the Holmes-Bonferroni correction. Sensitivity analysis used multiple imputations with a fully conditional specification (with 10 imputed datasets) to assess the impact of missing values.

\section{Results}

Incident data questionnaires were returned for 86 patients meeting the study inclusion criteria.

Seventy-one of 86 patients (82.6\%) presented to hospital eye services within 24 hrs of injury, with 83/86 
(96.6\%) presenting within 1 week of initial injury. Two patients presented more than 1 week after the initial injury, and the time elapsed between injury and presentation was not stated in one case. Patients demographics have previously been described in detail. ${ }^{17}$

Ocular trauma was unilateral in all reported cases. Trauma involving the globe was reported in 66/86 patients (76.7\%), of which 40/66 (60.1\%) were classified as openglobe. Eyelid trauma was reported in $26 / 86(30.2 \%)$ and orbital injury in 5/86 (5.8\%). Globe injuries were observed in a higher proportion of female patients than male patients in this cohort (globe injury in $25 / 27$ female patients compared to $41 / 58$ male patients; $p=0.0267$, gender not specified for one patient). Of the 40 open-globe injuries, the majority $(35 ; 87.5 \%)$ involved laceration of the cornea, with only 3 reported cases of scleral rupture (7.5\%). There was associated prolapse of uveal tissues in 25 cases $(62.5 \%)$ and vitreous incarceration in only one patient $(2.5 \%)$. Twenty-four of $86(27.9 \%)$ patients presented with a lid laceration, with canalicular involvement reported in $8 / 24$ patients (33.3\%).

At presentation, trauma to the anterior segment was reported in $57 / 86$ patients $(66.3 \%)$ and posterior segment injury was reported in 23/86 (26.7\%). Derangement of intraocular pressure (IOP) was observed in 22/86 (25.6\%) patients, with $9 / 22$ presenting with elevated IOP and 13/22 presenting with low IOP. All cases of elevated IOP were associated with hyphema. A detailed overview of clinical findings at presentation is illustrated in Table 1, and a summary of presenting injuries categorized according to the Birmingham Eye Trauma Terminology (BETT) system $^{18}$ is shown in Table 2 .

Overall, 15/86 (17.4\%) of patients had a best-corrected visual acuity (BCVA) of $6 / 6$ Snellen or better in the affected eye on presentation, 37/86 (43.0\%) had a BCVA of $6 / 24$ or better, $48 / 86(55.8 \%)$ had a BCVA of $6 / 60$ Snellen or better, and $25 / 86(29.1 \%)$ had a BCVA worse than $6 / 60$ Snellen. BCVA at presentation was not stated for 13 patients. Based on mid-2014 population statistics published by the Office for National Statistics (UK) ${ }^{19}$ as reported previously, ${ }^{17}$ these figures equate to incidence rates of 0.12 per 100,000 with visual acuity of $6 / 6$ or better, 0.29 per 100,000 better than $6 /$ 24, 0.37 per 100,000 better than $6 / 60$, and 0.19 per 100,000 worse than 6/60. Further breakdown of BCVA at presentation is represented graphically in Figure 1.
Table I Clinical findings at presentation

\begin{tabular}{|c|c|c|c|}
\hline Clinical finding & Description & $\mathbf{n}$ & $\% *$ \\
\hline Open-globe injury & $\begin{array}{l}\text { Any } \\
\text { Corneal laceration } \\
\text { Iris/uveal prolapse } \\
\text { Scleral rupture } \\
\text { Vitreous incarceration } \\
\text { Intraocular foreign body }\end{array}$ & $\begin{array}{l}40 \\
35 \\
25 \\
3 \\
1 \\
1\end{array}$ & $\begin{array}{l}46.5 \\
40.7 \\
29.1 \\
3.5 \\
1.2 \\
1.2\end{array}$ \\
\hline Eyelid trauma & $\begin{array}{l}\text { Any } \\
\text { Lid laceration } \\
\text { Lid foreign body } \\
\text { Canalicular injury } \\
\text { Eyelid burn } \\
\text { Eyelid ecchymosis }\end{array}$ & $\begin{array}{l}26 \\
24 \\
13 \\
8 \\
2 \\
1\end{array}$ & $\begin{array}{l}30.2 \\
27.9 \\
15.1 \\
9.3 \\
2.3 \\
1.2\end{array}$ \\
\hline $\begin{array}{l}\text { Anterior segment } \\
\text { trauma }\end{array}$ & $\begin{array}{l}\text { Any } \\
\text { Hyphema } \\
\text { Flat/shallow anterior } \\
\text { chamber } \\
\text { Traumatic cataract } \\
\text { Traumatic mydriasis } \\
\text { Angle recession } \\
\text { Iridodialysis } \\
\text { Corneal oedema } \\
\text { Corneal blood staining } \\
\text { Hypopyon }\end{array}$ & $\begin{array}{l}57 \\
24 \\
22 \\
13 \\
8 \\
4 \\
4 \\
2 \\
2 \\
1\end{array}$ & $\begin{array}{l}66.3 \\
27.9 \\
25.6 \\
15.1 \\
9.3 \\
4.7 \\
4.7 \\
2.3 \\
2.3 \\
1.2\end{array}$ \\
\hline $\begin{array}{l}\text { Posterior segment } \\
\text { trauma }\end{array}$ & $\begin{array}{l}\text { Any } \\
\text { Vitreous hemorrhage } \\
\text { No fundal view } \\
\text { Commotio retinae } \\
\text { Retinal detachment } \\
\text { Retinal tear } \\
\text { Choroidal rupture } \\
\text { Macular hole } \\
\text { Foveal laser burn } \\
\text { Submacular hemorrhage }\end{array}$ & $\begin{array}{l}23 \\
11 \\
8 \\
8 \\
5 \\
3 \\
3 \\
2 \\
1 \\
1\end{array}$ & $\begin{array}{l}26.7 \\
12.8 \\
9.3 \\
9.3 \\
5.8 \\
3.5 \\
3.5 \\
2.3 \\
1.2 \\
1.2\end{array}$ \\
\hline Trauma affecting IOP & $\begin{array}{l}\text { Any } \\
\text { Hypotony } \\
\text { Elevated IOP }\end{array}$ & $\begin{array}{l}22 \\
13 \\
9\end{array}$ & $\begin{array}{l}25.6 \\
15.1 \\
10.5\end{array}$ \\
\hline Orbital trauma & $\begin{array}{l}\text { Any } \\
\text { Orbital fracture } \\
\text { Traumatic optic } \\
\text { neuropathy } \\
\text { Orbital necrosis }\end{array}$ & $\begin{array}{l}5 \\
4 \\
2 \\
1\end{array}$ & $\begin{array}{l}5.8 \\
4.7 \\
2.3 \\
\\
1.2\end{array}$ \\
\hline Extraocular trauma & Facial soft tissue injury & 2 & 2.3 \\
\hline
\end{tabular}

Notes: Detailed breakdown ofclinical findings reported on incident questionnaires. $n=$ number of patients displaying each feature; $* \%$ shown as a proportion of whole sample. Many patients presented with multiple injuries in the same segment of the eye; number of injuries reported therefore exceeds total number of patients in the sample. Abbreviation: IOP, intraocular pressure. 
Table 2 Classification of injury at presentation.

\begin{tabular}{|l|l|l|l|}
\hline \multicolumn{2}{|l|}{ Classification of injury } & n & $\%$ \\
\hline $\begin{array}{l}\text { Adnexal injury only } \\
\text { Closed globe }\end{array}$ & & 23 & 26.7 \\
& Contusion & & \\
Open-globe & Lamellar laceration & 23 & 26.7 \\
& & 0 & 0 \\
& Penetrating & 34 & 39.5 \\
& IOFB & 1 & 1.2 \\
Total & Perforating & 1 & 1.2 \\
& Rupture & 4 & 4.7 \\
& & 86 & 100 \\
\hline
\end{tabular}

Note: Injury classified according to Birmingham Eye Trauma Terminology (BETT) system. ${ }^{18}$

Abbreviation: IOFB, intraocular foreign body.

Associations between presenting visual acuity and other presenting features and demographics are shown in Table 3. Non-white males presented with the best visual acuity whilst non-white females presented with the worst visual acuity $(p=0.005)$. Increasing severity of injury was associated with worse presenting visual acuity according to the BETT system $(p=0.006) .{ }^{18}$ There is weak evidence that increasing age was associated with improved presenting visual acuity in male, but not female patients $(p=0.009$ for the original dataset; $p=0.07$ for the imputed dataset). Neither time to presentation nor zone of injury added significant explanatory value to the model. Sensitivity analysis was performed to determine the extent to which the observed associations were sensitive to random variation in the missing data, by repeating the generalized linear model with multiple different imputations for missing values. Results were consistent with the primary analysis,

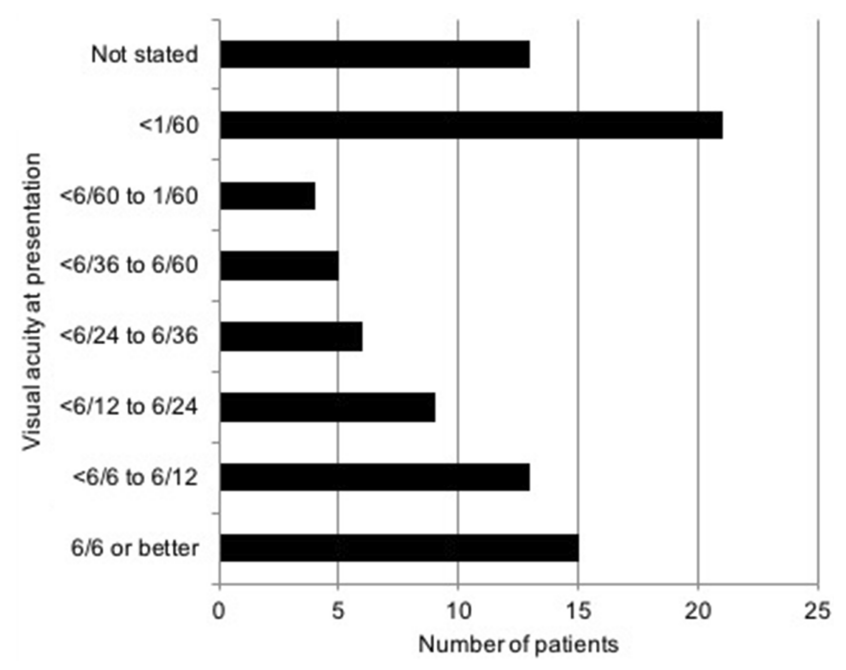

Figure I Best-corrected Snellen visual acuity at presentation. Data converted from LogMAR where appropriate. except for the effect of age, which did not maintain a statistically significant association with presenting visual acuity $(\log$ MAR +0.064 per female year; $p=0.289$, $\log$ MAR -0.12 per male year; $p=0.07)$.

Forty-seven of $86(54.7 \%)$ patients required examination under anesthesia (EUA) for detailed assessment of the extent of ocular trauma; EUA was performed within $24 \mathrm{hrs}$ of presentation in 35/47 (74.5\%) cases and within $72 \mathrm{hrs}$ in $41 / 47(87.2 \%)$ of cases. EUA was performed 4 days after presentation in 1 case, 6 days after presentation in 1 case, and greater than 7 days after presentation in 3 cases.

Surgical repair was necessary in 69/86 (80.2\%) cases, with this being performed within $24 \mathrm{hrs}$ of initial review (or EUA where first necessary) in 54/69 (78.3\%) cases.

\section{Discussion}

We describe presenting clinical features and initial management for 86 cases of ocular trauma affecting children aged 16 years or less in the UK, with data collected prospectively over a 12-month period through the BOSU reporting card scheme. In this sample, most patients presented to hospital eye services within $24 \mathrm{hrs}$ of initial injury $(82.6 \%)$, when EUA was necessary, it occurred within $24 \mathrm{hrs}$ in $74.5 \%$ of the cases, and when definitive surgical intervention was required, this was performed within $24 \mathrm{hrs}$ of initial assessment in the majority of cases $(78.3 \%)$.

Contextualizing our findings in the international literature describing rates of injury is a challenge because of the sparsity of robust data with tight disease definitions; authors have previously used the terms "penetrating" and "open" interchangeably, and the precise age range used to define a "pediatric" population has also been subject to variation.

MacEwen et al (1999) conducted a survey of childhood ocular trauma requiring hospital-admission for children aged 0-14 years over a 1-year period in Scotland, ${ }^{8}$ reporting 1 case of pediatric ocular trauma with visual acuity worse than $6 / 60$ Snellen. Using the population statistics on which this study was based, ${ }^{20}$ this equates to an incidence rate of 0.10 per 100,000 population ( $0-14$ years, Scotland only) (95\% confidence interval $0.02-0.60$ per 100,000$)$. We report 25 cases with vision less than $6 / 60$ at presentation, indicating a current incidence rate of 0.19 cases per 100,000 population ( $0-16$ years, entire UK) $(95 \%$ confidence interval $0.13-0.29$ per 100,000$)$. This suggests that there has been no reduction in the rate of severe pediatric ocular trauma over the past 25 years. 
Table 3 Factors influencing presenting visual acuity $(\mathrm{VA})^{18}$

\begin{tabular}{|l|l|l|}
\hline Predictors & Difference in logMAR VA & 95\% confidence interval \\
\hline White female & Reference category & \\
Non-white female & 1.43 & +0.52 to +2.35 \\
White male & 0.78 & -0.21 to +1.78 \\
Non-white male & -1.66 & -2.82 to -0.50 \\
Adnexal injury only & Reference category & \\
Contusion & 0.73 & +0.16 to +1.30 \\
Penetrating or IOFB & 0.53 & +0.02 to +1.04 \\
Perforating or rupture & 1.38 & +0.53 to +2.24 \\
$+I$ year age in female patients & +0.13 & +0.03 to +2.32 \\
$+I$ year age in male patients & -0.16 & -0.04 to -2.71 \\
\hline
\end{tabular}

Notes: Results of a generalized linear model created to assess the effect of time to presentation, gender, ethnicity, age, time to surgery, and injury classification according to BETT. ${ }^{18}$ Nonsignificant variables were removed from the model, and response categories collapsed where appropriate to improve model fit.

Abbreviation: IOFB, intraocular foreign body.

Our data demonstrate a higher proportion of open injuries $(60.1 \%)$ and greater proportion of patients requiring surgical intervention (80.2\%) than previously reported within the UK population or within population-based studies from other developed countries. In the previously described Scottish study, ${ }^{8}$ penetrating injury was observed in only $24 \%$ of the patients (22/93) compared to $46.5 \%$ (40/86) with open injury in the present study, and surgical intervention required in $48 \%$ compared to $80.2 \%$ in our series. Furthermore, Strahlman et al (1990) showed only $16 \%$ of hospitalizations in Maryland, USA, were for penetrating injuries, ${ }^{21}$ and a similar survey in Western Australia reported open injuries in $72 / 482$ or $14.9 \%$ of the cases. ${ }^{9}$

Despite the higher proportion of open injuries admitted to UK hospitals in our study, population-based estimates suggest that the incidence rate of open/penetrating ocular injury is lower than previously reported. MacEwen et al reported 22 cases of penetrating trauma in their Scottish population, ${ }^{8}$ which equates to an incidence rate of 2.29 per $100,000$ (95\% confidence interval $1.51-3.47$ per 100,000$)$. Strahlman et al report an incidence rate of open injury of 3.9 per 100,000 (95\% confidence interval $2.1-5.8$ per $100,000) .{ }^{21}$ Based on population estimates for our data, we report a rate of open injury of 0.31 per 100,000 (95\% confidence interval $0.23-0.42$ per 100,000 ).

We therefore describe a reduced incidence of open injuries overall, but an increased proportion of children admitted due to open injuries requiring surgery and find no evidence of improvement in presenting acuity. This is likely to reflect a shift in practice with regard to the management of pediatric ocular trauma; it is now common in the UK for all but the most serious cases of ocular trauma to be managed on an out-patient basis, whereas a greater proportion of patients may have been admitted for inpatient care at the time of the previous investigation. For example, in the previous series, $60 \%$ of the cases were admitted for hyphema management; in comparison, only $28 \%$ had a hyphema in the present series, which may have been an adjunctive finding rather than the primary reason for admission.

Corneal laceration was the most commonly reported clinical finding in our sample, being documented in $40.7 \%$ of the patients, followed by lid laceration, which was observed in $27.9 \%$ of the patients. These figures are in contrast to a recent similar large-scale study of pediatric ocular trauma in Finland: The Helsinki Eye Trauma Study collected data on ocular injuries in patients aged 16 years or less presenting to a single tertiary referral eye hospital over a one-year period. ${ }^{22}$ This study reported contusion as the most common diagnosis, being observed in $62 / 202$ (30.7\%) patients, and reported open-globe injury in only $6 / 202(3.0 \%)$ patients. The researchers reported only $18 /$ 202 cases of lid laceration (8.9\%). The comparably high severity of ocular injury described in our study is reflected in the presenting levels of visual acuity; $29.1 \%$ of the patients in our sample were documented as achieving a BCVA of worse than $6 / 60$ in the affected eye at presentation, compared to $6.9 \%$ in the Helsinki Eye Trauma Study. The reasons for this difference in reported injury severity are likely to be due to our sampling criteria: Haavisto et al reported outcomes for all cases ocular trauma presenting to the emergency clinic, whilst our inclusion criteria required ophthalmologists to report only cases of pediatric ocular trauma requiring day-case or overnight admission. As a result, ocular trauma which did not require admission is not included in our series, and our data therefore reflect 
more serious cases of ocular trauma. Furthermore, the mechanisms of ocular injury causing presentation in Helsinki differ to the UK; in the Helsinki, the commonest single mechanism of injury was a snowball projectile, ${ }^{23}$ which was not found to cause any presentation in our series.

Thompson et al reported on the etiology and mechanism of open-globe injuries affecting 72 children over a 6year period in Sydney, Australia (2002). ${ }^{24}$ Results were similar; comparing our data with Sydney, the majority of penetrating injuries involved corneal laceration (41\% vs $58 \%$ ), with a high rate of uveal prolapse (25\% vs $68 \%$ ) and a relatively low incidence of scleral laceration (4\% vs $13 \%$ ). The majority of injuries in this cohort were lacerations caused by sharp objects $(45 / 72)$.

As would be expected, we demonstrate that presenting visual acuity deteriorates with increasing severity of ocular injury; children with perforating injuries or ruptures present with worse vision than those with closed globe or adnexal injuries only. Surprisingly, there was no evidence of a difference in the presenting acuity between patients with closed, penetrating and perforating injuries or ruptures. This may suggest that presenting acuity in children is strongly modulated by factors other than injury severity or may be attributable to the small number of children with perforating injuries or ruptures in our sample, resulting in a lack of power to detect a significant association with presenting acuity in our generalized linear model.

Our data also suggest there may be variation in presenting visual acuity following ocular injury between children of different gender and ethnicity, although these associations appear complex, with non-white males presenting with better vision, and non-white females presenting with worse vision in our sample. The effect of age may also be modulated by gender, with a trend for increasing presenting acuity with increasing age in males only.

It is difficult to explain these associations; the improvement in presenting acuity seen with increasing age amongst males may suggest that males may become more cautious as they mature, engaging in relatively higher-risk behaviour at a younger age, but becoming more aware of potential risks in later childhood. The lack of similar association in female children may imply that age has less influence on risktaking behaviour in this cohort. Equally, it is possible that males and females react differently to injury and stress stimuli. Further research is required to explain these observations.
It is worth noting that whilst presenting visual acuity is strongly affected by injury severity, ${ }^{18}$ other cultural factors and the approach taken by the assessing clinician may also influence presentation.

Examination under anesthesia was commonly performed (in $54.7 \%$ of the patients) highlighting the difficulty in examining this patient group. In one case, the diagnosis of open-globe injury was missed - even on EUA. The frequency of elevated IOP (25\%) underlines the importance of thorough assessment with adequate posterior segment examination and reliable IOP measurement in all cases.

Taken collectively these observations have a number of key implications:

First, children in the UK population may be subject to increasing severity of ocular trauma. We have previously reported on the high incidence of trauma due to thrown objects $^{17}$ and would therefore argue that the majority of penetrating trauma in children is preventable. Our findings show that more needs to be done to prevent such injury.

Second, eye-care providers must be able to provide the necessary services for assessment and management of severe pediatric ocular trauma in the acute emergency setting. The Royal College of Ophthalmologists (UK) provides limited guidance for pediatric emergency services $^{25}$ but suggests that all ophthalmic units should have recognized emergency cover arrangements with access to pediatric advice and facilities, and that "anterior segment surgery in children should be carried out by consultants with appropriate specialist training and expertise". We would recommend the development of specific guidelines for the provision of pediatric ocular trauma services and the delivery of these services within clear prospective care pathways, key components of which are pediatric anesthesia, pediatric ophthalmologists, and specialists able to deliver vitreoretinal, anterior-segment, and oculoplastic surgery in children where appropriate.

Third, ocular injury in children is very different to that in adults. A significant proportion of open-globe injury in adults are globe ruptures, being strongly associated with falls in older age groups, ${ }^{26}$ whilst we demonstrate more lacerations from sharp objects in the pediatric population. In addition, the high probability of reduced vision even in patients without eye injuries and the difficulty of distinguishing closed globe from open-globe injuries mean that assessment and consequent appropriate management are more challenging in pediatric practice. Occult globe injury in children may be missed by the less experienced 
clinician, as demonstrated in one child of age 16 months who fell onto a shattered ceramic pot and in whom openglobe injury was missed under EUA and at two different hospitals.

This prospective cross-sectional review is subject to a number of limitations. First, the BOSU reporting card scheme depends on accurate data from the cohort of consultant ophthalmologists in the UK and may miss new appointments or those who have changed location. Second, ophthalmologists may not keep track of reported patients and their hospital identification at the initial stage of reporting, resulting in subsequent difficulty tracing the patient; strategies to minimize this loss of data have been described in detail in a previous publication. ${ }^{16}$ Finally, clinicians are often under severe pressure and may not have the time to engage with BOSU or complete data collection proformas as requested. Further work is in progress to determine the rate of potential unreported ocular trauma during the course of our initial investigation.

Whilst our findings suggest that pediatric ocular trauma is being appropriately assessed and managed throughout the UK, we are reminded that prevention remains far better than cure. Ultimately, we believe that interventions aimed at avoiding unnecessary injury are far more effective at preventing visual loss than any form of medical or surgical intervention following injury. We have previously advocated for the use of appropriate eye protection for children engaging in "high-risk" activities, and increased education for those supervising children in domestic and play environments. ${ }^{17}$ Through dissemination of the results of the UK Paediatric Ocular Trauma Study, we hope to improve both primary prevention of injury and secondary care for affected children in the UK and beyond.

\section{Abbreviations list}

BETT, Birmingham Eye Trauma Terminology; BCVA, bestcorrected visual acuity; BOSU, British Ophthalmological Surveillance Unit; EUA, examination under anesthesia; IOFB, intraocular foreign body; IOP, intraocular pressure; POTS, Paediatric Ocular Trauma Study; UK, United Kingdom.

\section{Ethics approval}

This study was approved by the Cambridge South Research Ethics Committee, Cambridge, UK. Research and developmentapproval was granted by Birmingham
Children's Hospital Research and Development Department, Birmingham, UK.

\section{Data availability}

Data supporting the results reported in this manuscript are available from the corresponding author on request.

\section{Acknowledgments}

The authors wish to thank the Royal College of Ophthalmologists British Ophthalmic Surveillance Unit (BOSU), and in particular, Mr Barny Foot for his advice at various stages of this project's inception. We also acknowledge the BOSU review committee members for their contribution to the design of POTS data collection questionnaires. This study was supported by a bursary from the British Ophthalmological Surveillance Unit (BOSU).

\section{Author contributions}

All authors contributed towards data analysis, drafting and critically revising the paper, gave final approval of the version to be published, and agreed to be accountable for all aspects of the work.

\section{Disclosure}

The authors report no conflicts of interest in regard to this work.

\section{References}

1. Abbott J, Shah P. The epidemiology and etiology of pediatric ocular trauma. Surv Ophthalmol. 2013;58:476-485. doi:10.1016/j. survophthal.2012.10.007

2. May DR, Kuhn FP, Morris RE, et al. The epidemiology of serious eye injuries from the United States Eye Injury Registry. Graefes Arch Clin Exp Ophthalmol. 2000;238:153-157. doi:10.1007/PL00007884

3. Négrel AD, Thylefors B. The global impact of eye injuries. Ophthalmic Epidemiol. 1998;5:143-169. doi:10.1076/opep.5.3. 143.8364

4. Rahi JS, Cumberland PM, Peckham CS. Visual function in workingage adults: early life influences and associations with health and social outcomes. Ophthalmology. 2009;116:1866-1871. doi:10.1016/j. ophtha.2009.03.007

5. Agrawal R, Shah M, Mireskandari K, Yong GK. Controversies in ocular trauma classification and management: review. Int Ophthalmol. 2013;33:435-445. doi:10.1007/s10792-012-9698-y

6. Gupta A, Rahman I, Leatherbarrow B. Open globe injuries in children: factors predictive of a poor final visual acuity. Eye (Lond). 2009;23:621-625. doi:10.1038/eye.2008.32

7. Brophy M, Sinclair SA, Hostetler SG, Xiang H. Pediatric eye injury related hospitalizations in the United States. Paediatrics. 2006;117(6): e1263-e1271. doi:10.1542/peds.2005-1950

8. MacEwen C, Baines P, Desai P. Eye injuries in children: the current picture. Br J Ophthalmol. 1999;83:933-936. doi:10.1136/bjo.83.8.933 
9. Yardley A-ME, Hoskin AK, Hanman K, Sanfilippo PG, Lam GC, Mackey DA. Paediatric ocular and adnexal injuries requiring hospitalisation in Western Australia. Clin Exp Optom. 2017;100:227-233. doi:10.1111/cxo.12486

10. Kadappu S, Silveira S, Martin F. Aetiology and outcome of open and closed globe eye injuries in children. Clin Exp Ophthalmol. 2013;41:427-434. doi:10.1111/ceo.12034

11. Podbielski DW, Surkont M, Tehrani NN, Ratnapalan S. Pediatric eye injuries in a Canadian emergency department. Can J Ophthalmol. 2009;44:519-522. doi:10.3129/109-093

12. Pizzarello LD. Ocular trauma: time for action. Ophthalmic Epidemiol. 1998;5:115-116. doi:10.1076/opep.5.3.115.8366

13. Shah A, Blackhall K, Ker K, Patel D. Educational interventions for the prevention of eye injuries. In: Shah A, editor. Cochrane Database of Systematic Reviews. Chichester (UK): John Wiley \& Sons, Ltd; 2009:CD006527.

14. Philip SS, Hoskin AK. Children's protective eyewear: the challenges and the way forward. Med J Aust. 2014;201:87-88. doi:10.5694/ mja13.00132

15. Hoskin AK, Philip SS, Yardley A-ME, Mackey DA. Eye injury prevention for the pediatric population. Asia-Pacific J Ophthalmol. 2016;5:202-211. doi:10.1097/APO.0000000000000193

16. Sii F, Barry R, Blanch R, Abbott J, MacEwen CJ, Shah P. The UK Paediatric Ocular Trauma Study 1 (POTS1): development of a global standardized protocol for prospective data collection in pediatric ocular trauma. Clin Ophthalmol. 2017;11:449-452. doi:10.2147/ OPTH.S125160

17. Sii F, Barry RJ, Abbott J, Blanch RJ, MacEwen CJ, Shah P. The UK Paediatric Ocular Trauma Study 2 (POTS2): demographics and mechanisms of injuries. Clin Ophthalmol. 2018;12:105-111. doi:10.2147/OPTH.S155611
18. Khun F, Morris R, Witherspoon CD. Birmingham Eye Trauma Terminology (BETT): terminology and classification of mechanical eye injuries. Ophthalmol Clin North Am. 2002;15(2):139-143.

19. Office for National Statistics. Population Estimates Analysis Tool [Internet]. Available from: https://www.ons.gov.uk/peoplepopulatio nandcommunity/populationandmigration/populationestimates/data sets/populationestimatesanalysistool. Accessed August 7, 2017.

20. Desai P, MacEwen CJ, Baines P, Minassian DC. Incidence of cases of ocular trauma admitted to hospital and incidence of blinding outcome. $\mathrm{Br} J$ Ophthalmol. 1996;80(7):592-596. doi:10.1136/ bjo.80.7.592

21. Strahlman E, Elman M, Daub E, Baker S. Causes of pediatric eye injuries. A population-based study. Arch Ophthalmol. 1990;108 (4):603-606. doi:10.1001/archopht.1990.01070060151066

22. Haavisto A-K, Sahraravand A, Holopainen JM, Leivo T. Paediatric eye injuries in Finland - Helsinki eye trauma study. Acta Ophthalmol. 2017;95:392-399. doi:10.1111/aos.13273

23. Niiranen M, Raivio I. Eye injuries in children. The Br J Ophthalmol. 1981;65(6):436. doi:10.1136/bjo.65.8.553

24. Thompson CG, Kumar N, Billson FA, Martin F. The aetiology of perforating ocular injuries in children. $\mathrm{Br} J$ Ophthalmol. 2002;86:920-922. doi:10.1136/bjo.86.8.920

25. Royal College of Ophthalmologists UK ophthalmic services guidance: ophthalmic services for children 2012. Available from: https://www.rcophth.ac.uk/wp-content/uploads/2014/12/ 2012_PROF_182_Ophthalmic-Services-for-Children.pdf. Accessed February 10, 2018.

26. Beshay N, Keay L, Dunn H, Kamalden TA, Hoskin AK, Watson SL. The epidemiology of open globe injuries presenting to a tertiary referral eye hospital in Australia. Inj Int $J$ Care Injured. 2017;48:1348-1354. doi:10.1016/j.injury.2017.04.035
Clinical Ophthalmology

\section{Publish your work in this journal}

Clinical Ophthalmology is an international, peer-reviewed journal covering all subspecialties within ophthalmology. Key topics include: Optometry; Visual science; Pharmacology and drug therapy in eye diseases; Basic Sciences; Primary and Secondary eye care; Patient Safety and Quality of Care Improvements. This journal is indexed on PubMed

\section{Dovepress}

Central and CAS, and is the official journal of The Society of Clinical Ophthalmology (SCO). The manuscript management system is completely online and includes a very quick and fair peer-review system, which is all easy to use. Visit http://www.dovepress.com/ testimonials.php to read real quotes from published authors. 